\title{
Supplement to: Statistical Modeling and Analysis of Trace Element Concentrations in Forensic Glass Evidence
}

\author{
Karen D. H. Pan*,‡, Karen Kafadar ${ }^{\dagger, \ddagger}$ \\ University of Virginia
}

\section{Introduction}

This supplement contains additional match rate plots for simulations using the German and Canadian covariance matrices from Section 6.1 and 6.2. These simulations used a covariance matrix $V^{*}=V_{e}^{*}+V_{f}^{*}$ that takes into account both between and within fragment variability. All figures in this document will consider only measurement variability, $V_{e}^{*}$. An overall decrease in match rates can be seen.

\section{German and Canadian covariance simulations}

Section 6.1 omitted robust correlation matrices calculated using fast MCD as they were very similar to the classical correlation matrices; the difference is larger when only considering measurement variability. Figure 1 shows these correlation matrices and Figure 2 shows corresponding match rates.

\footnotetext{
*Partial support from National Institute of Standards \& Technology via subcontract from Iowa State University

${ }^{\dagger}$ Isaac Newton Institute for Mathematical Sciences, Probability and Statistics in Forensic Science, EPSRC Grant Number EP/K032208/1.
} 

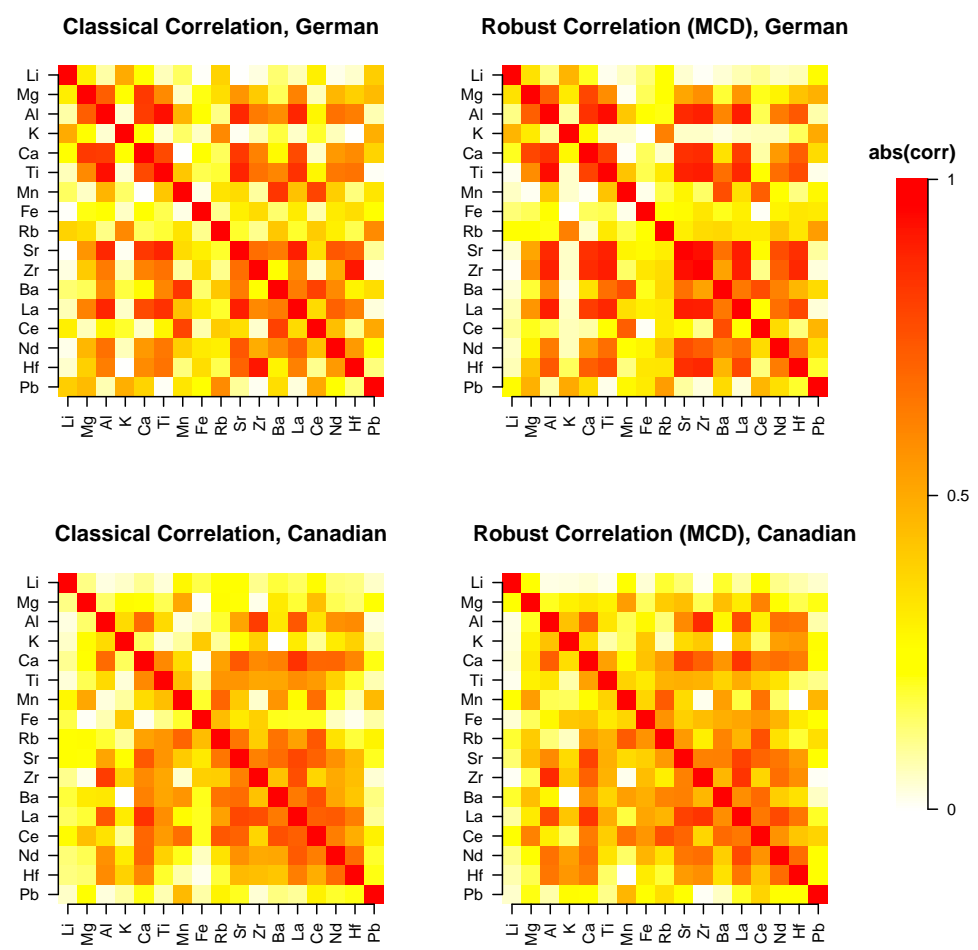

Figure 1: (Analogous to paper Figure 1) Classical and robust correlation matrices for the German and Canadian data sets using $V_{e}^{*}$. The discrepancy between robust and classical correlations is slightly larger here.

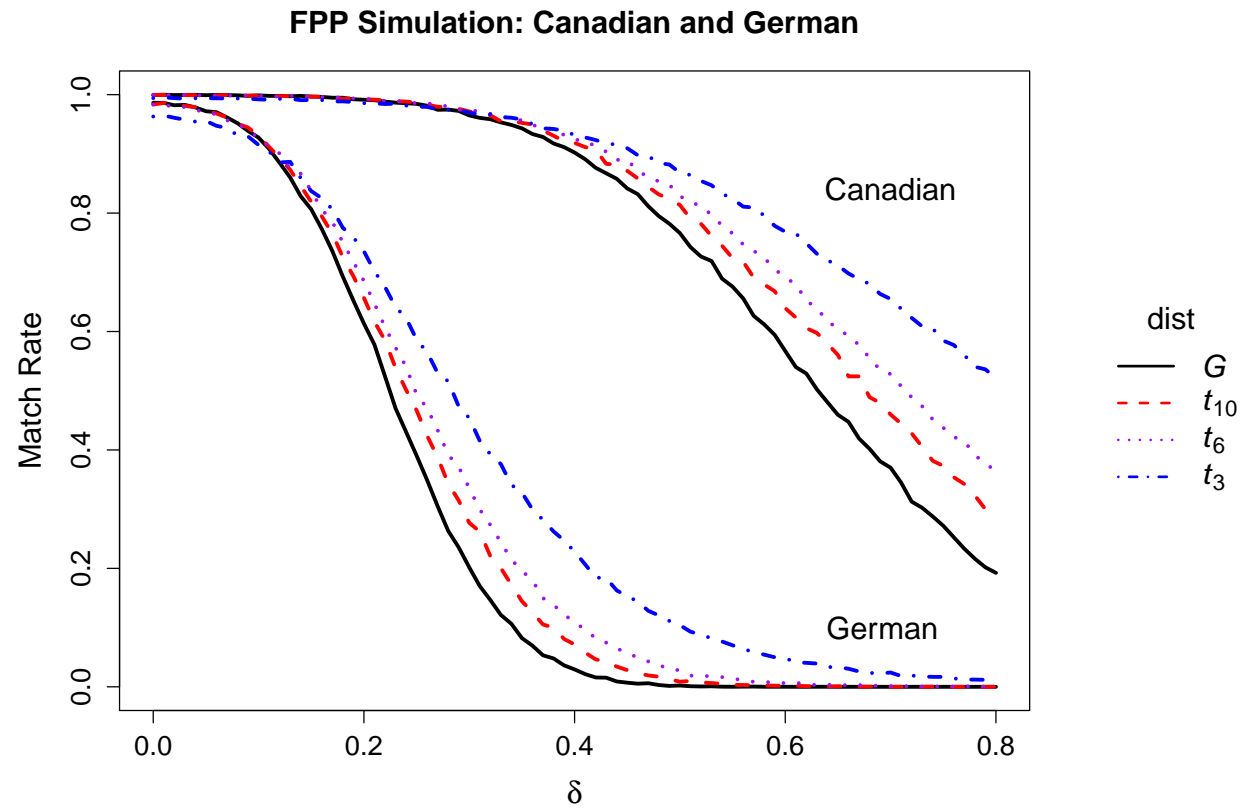

Figure 2: (Analogous to paper Figure 2) Match rates from Canadian and German simulations for data from four different distributions. $\delta$ gives the approximate relative change in means. The match rates are considerably lower as $\delta$ increases in comparison to $V^{*}=V_{e}^{*}+V_{f}^{*}$. 


\section{Canadian covariance matrix simulations}

As mentioned in Section 6.2, we noticed large set-to-set variation in the concentrations of ${ }^{29} \mathrm{~K}$ and ${ }^{57} \mathrm{Fe}$, possibly due to (1) the ubiquity of these elements in the surrounding environment which may be present at different levels on different days resulting in varying levels of contamination; and (2) these tend to be badly behaved elements due to interference from the plasma. However as both elements remain listed in standard ASTM E2926-16, we simulated error rates using both $p=17$ and $p=15$ elements (all but ${ }^{39} \mathrm{~K}$ and ${ }^{57} \mathrm{Fe}$ ). The figures below allow for comparisons between the two. These figures use covariance $V^{*}=V_{e}^{*}$, but the effect of $p$ is similar when fragment variability is included.
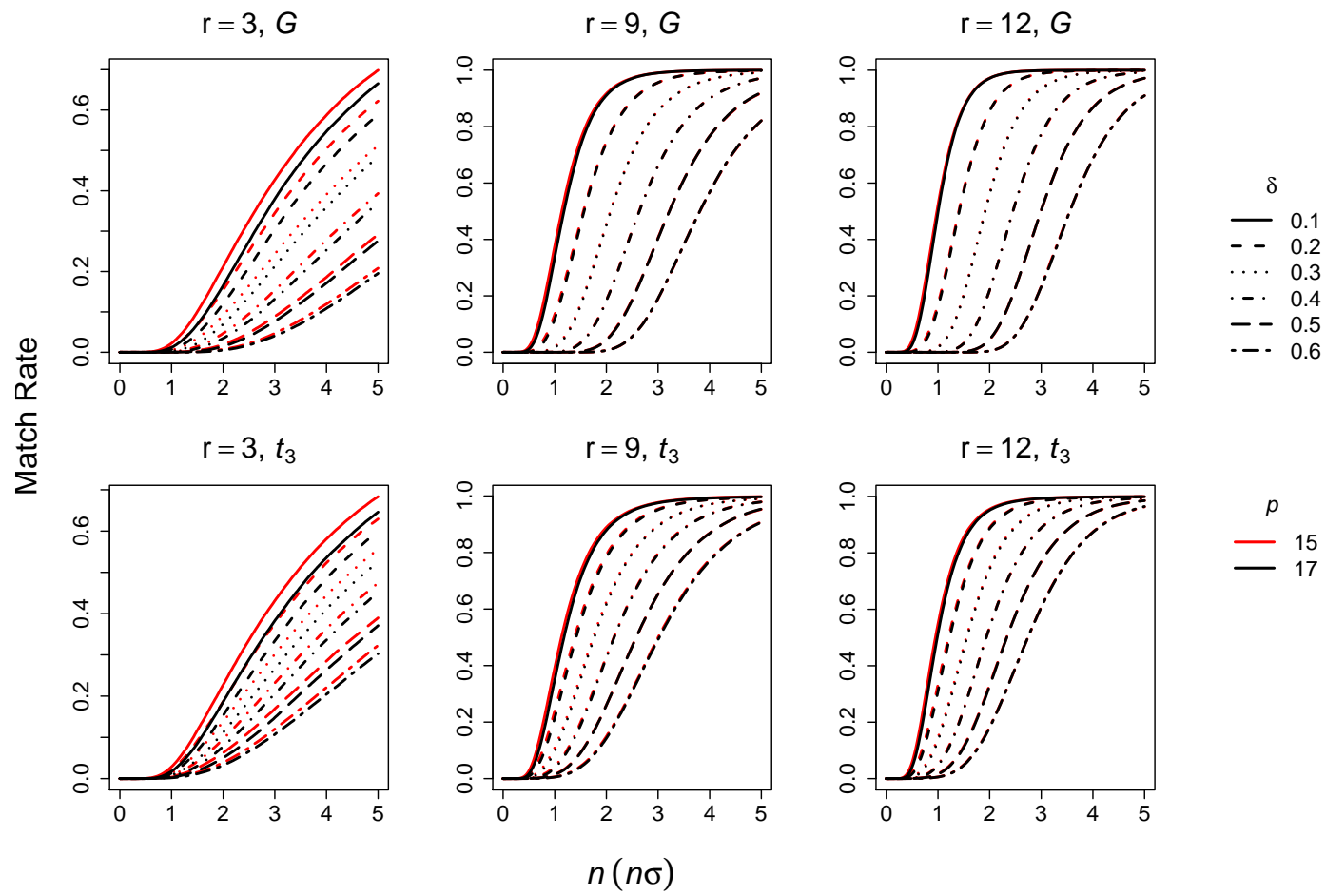

Figure 3: (Analogous to paper Figure 3) Simulation match rates using the Canadian covariance matrix by sample size and distribution for six $\delta$ values with $n=0, \ldots, 5$, shown for $p=15$ and $p=17$.

\begin{tabular}{lrrrrrr}
\hline$(\delta)$ & 0.1 & 0.2 & 0.3 & 0.4 & 0.5 & 0.6 \\
\hline $3 G$ & 0.547 & 0.467 & 0.359 & 0.253 & 0.171 & 0.109 \\
$3 t_{3}$ & 0.535 & 0.485 & 0.411 & 0.336 & 0.264 & 0.204 \\
$9 G$ & 0.999 & 0.992 & 0.967 & 0.900 & 0.767 & 0.568 \\
$9 t_{3}$ & 0.992 & 0.987 & 0.970 & 0.935 & 0.868 & 0.771 \\
$12 G$ & 1.000 & 0.999 & 0.992 & 0.962 & 0.867 & 0.687 \\
$12 t_{3}$ & 0.998 & 0.996 & 0.992 & 0.977 & 0.941 & 0.873 \\
\hline
\end{tabular}

Table 1: (Analogous to paper Table 4) Match rates for Figure 3 by sample size at $n=4$ and $p=17\left(G=\right.$ Gaussian, $t_{3}=t$ with $\left.\mathrm{df}=3\right)$ for various $\delta$. 
The next set of figures compares match rates for Hotelling's $T^{2}$, the 4-SD approach, and several $t$ confidence intervals. Figures 4 and 6 here are analogous to Figures 4 and 5 in the paper, and Figure 5 here compares match rates from Hotelling's $T^{2}$ under different distributions and sample sizes. Match rates for the 4-SD approach using $p=15$ are very similar to $p=17$, and are omitted. We note that incorporating more variability (adding $V_{f}^{*}$ to $V_{e}^{*}$ ) results in increasing error rates for the 4-SD approach (and any $t$ confidence intervals) as expected since more variability is accounted for, but results in decreasing error rates for Hotelling's $T^{2}$. This may be because including within fragment variability considerably increases the correlations between elements, especially for the Canadian covariance matrix, on which these simulations are based (see Figure 1 here versus Figure 1 in paper).

Figures 7 and 8 show in more detail match rate curves between $p=15$ and $p=17$ from Figure 6.

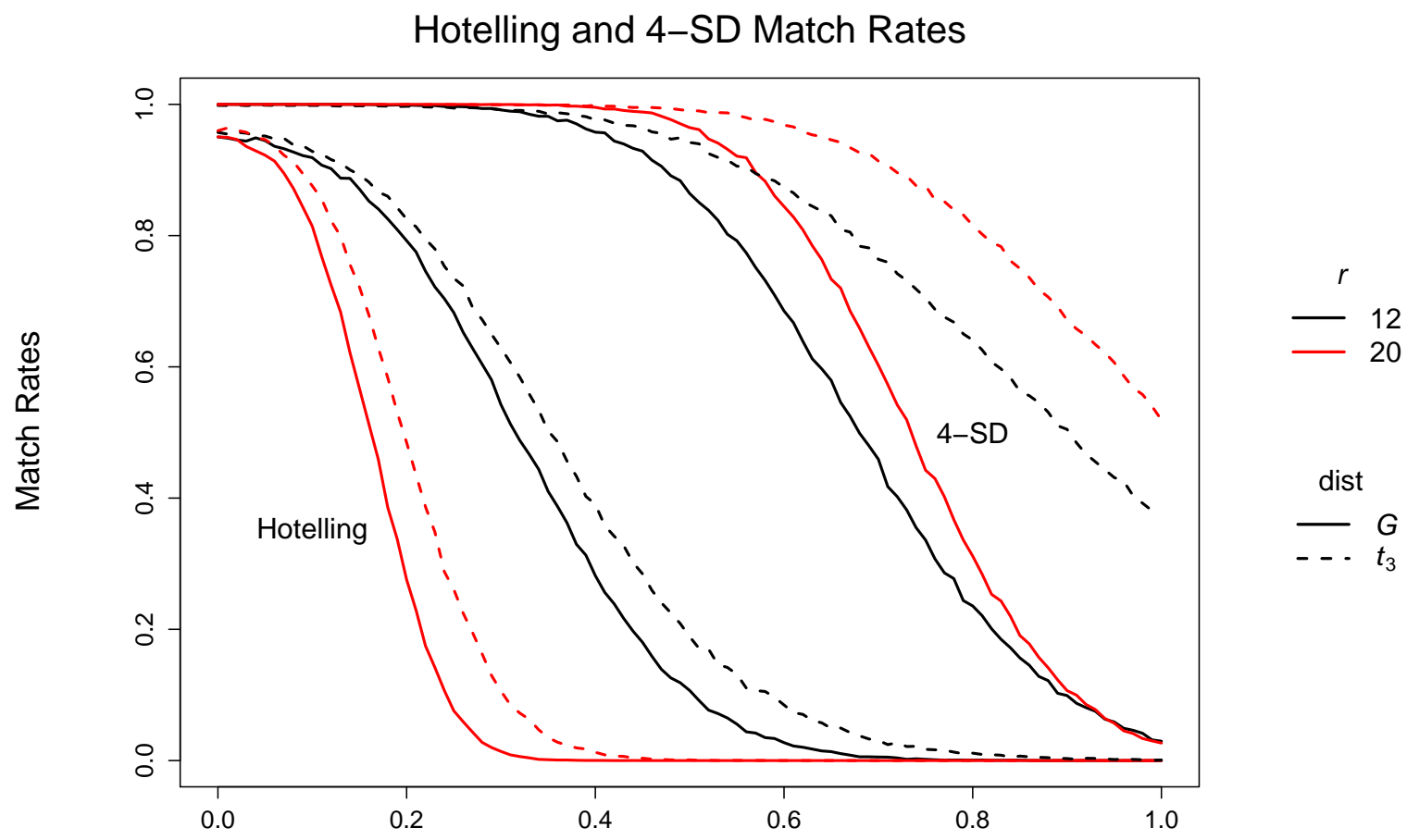

$\delta$

Figure 4: (Analogous to paper Figure 4) Match rates for Hotelling's $T^{2}$ vs. 4-SD approach for $G$ and $t_{3}$ distributed data. Distribution has a larger effect on the 4-SD approach. 


\section{Hotelling Match Rates}

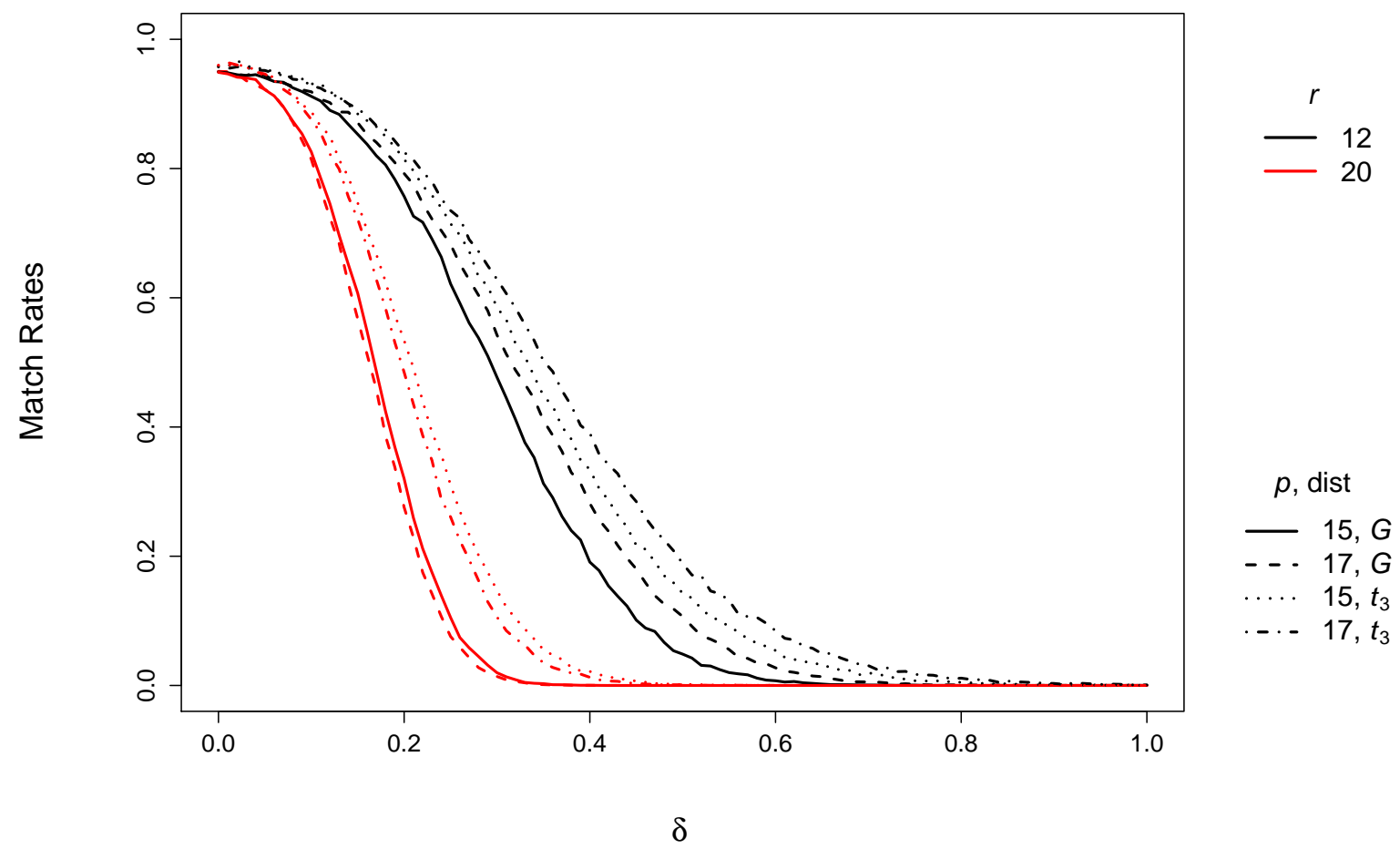

Figure 5: Match rates for Hotelling's $T^{2}$ comparing $p=15$ and $p=17$ for $G$ and $t_{3}$ data. Match rates from the 4-SD approach are very similar for both $p$ and are not shown.

Hotelling and $t$ Confidence Intervals, $G$

Hotelling and $t$ Confidence Intervals, $t_{3}$
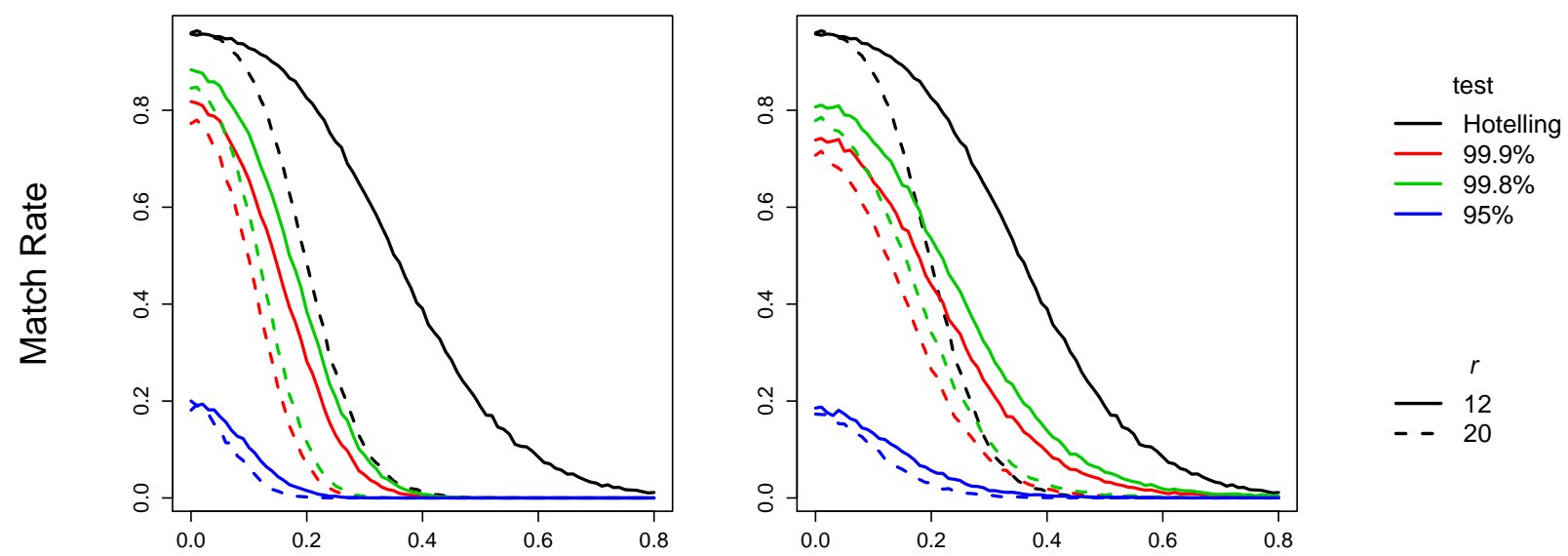

$\delta$

Figure 6: (Analogous to paper Figure 5) Match rates for Hotelling's $T^{2}$ and $t$ (95\%, 99.8\%, $99.9 \%)$ confidence intervals. 


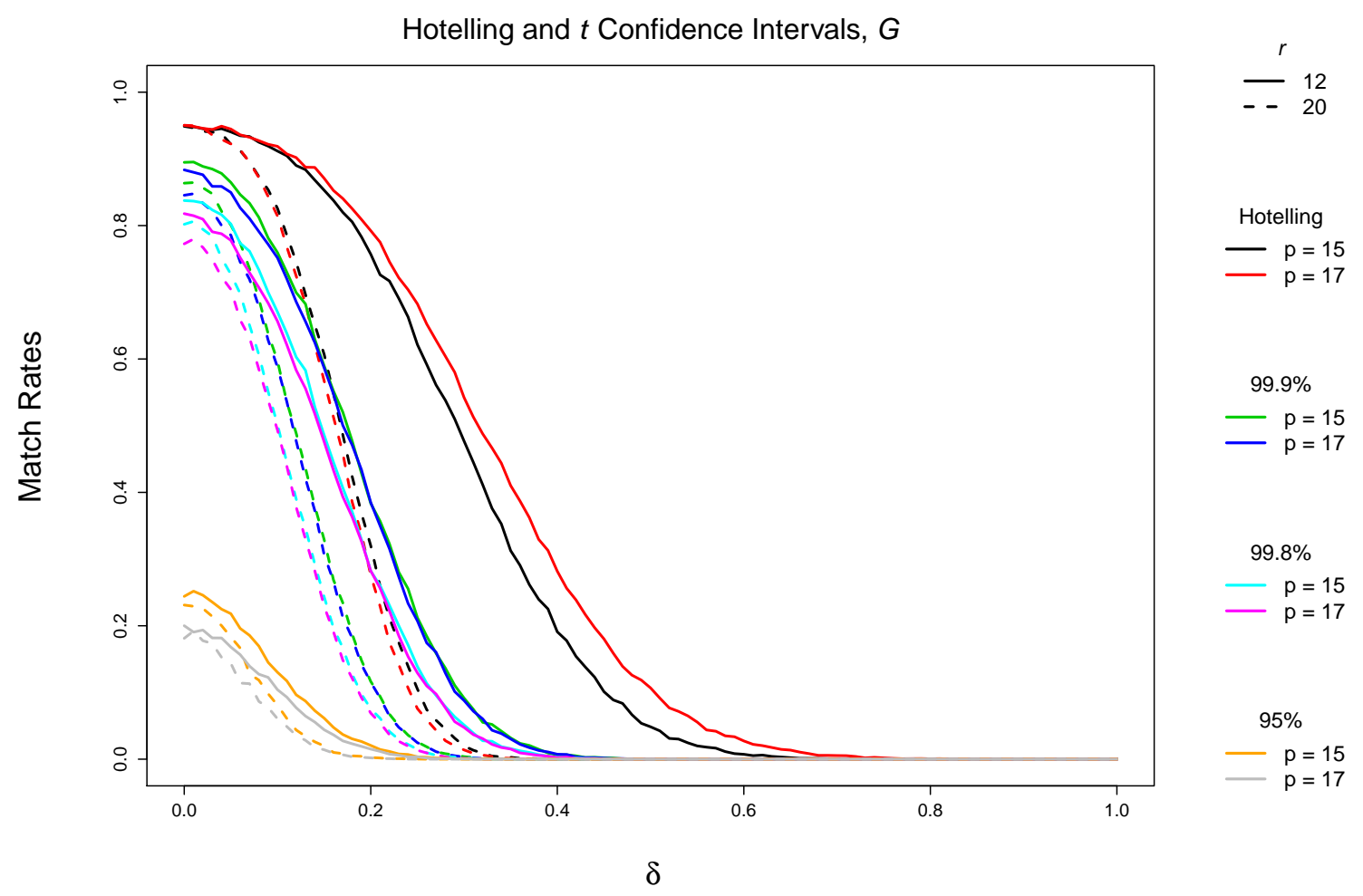

Figure 7: Hotelling's $T^{2}$ and $t$ confidence interval match rates for $G$ data.

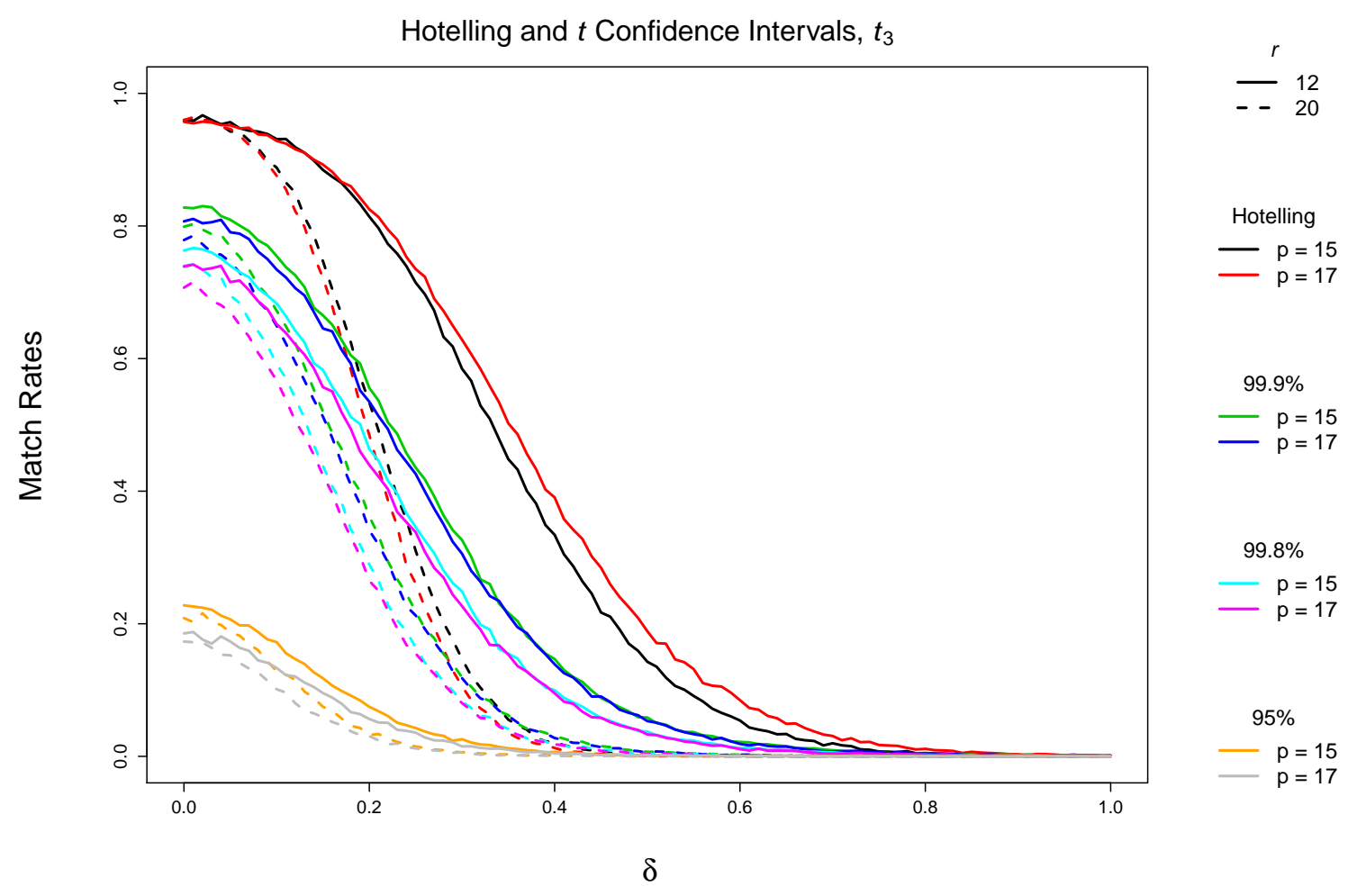

Figure 8: Hotelling's $T^{2}$ and $t$ confidence interval match rates for $t_{3}$ data. 


\section{Acknowledgements}

We are grateful to Dr. David Ruddell, Centre for Forensic Science, Toronto, for sharing his laboratory's data and for many helpful discussions throughout our project. We also thank Dr. Peter Weis, Bundeskriminalamt/Federal Criminal Police Office, Forensic Science Institute, Wiesbaden, Germany, for providing an electronic version of data published in Weis et al. (2011). The FIU data were obtained from the Technical Support Working Group via Jeff Huber (jeff.huber.ctr@cttso.gov). We also are grateful to the anonymous reviewers who provided useful feedback that led to improvements in this article. The second author also thanks the Isaac Newton Institute for Mathematical Sciences, Cambridge U.K., for its hospitality during the program Probability and Statistics in Forensic Science which was supported by EPSRC Grant Number EP/K032208/1. Finally, we gratefully acknowledge the late Stephen Fienberg, whose life-long commitment to rigorous science in many disciplines of great public importance, including those arising in criminal justice and forensic science, inspired us to pursue this work.

\section{References}

[1] ASTM International. (2012). ASTM E2330-12 Standard Test Method for Determination of Concentrations of Elements in Glass Samples Using Inductively Coupled Plasma Mass Spectrometry (ICP-MS) for Forensic Comparisons. Retrieved from https://doi.org/10.1520/E2330-12

[2] ASTM International. (2013). ASTM E2926-13 Standard Test Method for Forensic Comparison of Glass Using Micro X-ray Fluorescence ( $\mu$-XRF) Spectrometry. Retrieved from https://doi.org/10.1520/E2926

[3] ASTM International. (2016). ASTM E2927-16e1 Standard Test Method for Determination of Trace Elements in Soda-Lime Glass Samples Using Laser Ablation Inductively Coupled Plasma Mass Spectrometry for Forensic Comparisons. Retrieved from https://doi.org/10.1520/E2927-16E01

[4] Dorn, H., Ruddell, D. E., Heydon, A., Burton, B. D. (2015). Discrimination of float glass by LA-ICP-MS: assessment of exclusion criteria using casework samples. Canadian Society of Forensic Science Journal 48(2): 85-96 (doi: 10.1080/00085030.2015.1019224).

[5] Weis, P., Dückling, M., Watzke, P., Menges, S., Becker, S. (2011). Establishing a match criterion in forensic comparison analysis of float glass using laser ablation inductively coupled plasma mass spectrometry, Journal of Analytical Atomic Spectroscopy 26:1273-1284. 